\title{
The C-Type Lectin Receptor Mincle Binds to Streptococcus pneumoniae but Plays a Limited Role in the Anti-Pneumococcal Innate Immune Response
}

\author{
Anne Rabes ${ }^{1 \ddagger}$, Stephanie Zimmermann ${ }^{2,3 \ddagger}$, Katrin Reppe ${ }^{1}$, Roland Lang ${ }^{4}$, \\ Peter H. Seeberger ${ }^{2}$, Norbert Suttorp ${ }^{1}$, Martin Witzenrath ${ }^{1}$, Bernd Lepenies ${ }^{2,3 *}$, \\ Bastian Opitz ${ }^{1 *}$
}

1 Department of Internal Medicine/Infectious Diseases and Pulmonary Medicine, Charité Universitätsmedizin Berlin, Berlin, Germany, 2 Max Planck Institute of Colloids and Interfaces, Department of Biomolecular Systems, Potsdam, Germany, 3 Freie Universität Berlin, Institute of Chemistry and Biochemistry, Department of Biology, Chemistry and Pharmacy, Berlin, Germany, 4 Institute for Clinical Microbiology, Immunology and Hygiene, University Hospital Erlangen, Erlangen, Germany

จ These authors are joint senior authors on this work.

$\ddagger$ These authors are first authors for this work.

* bastian.opitz@ charite.de (BO); bernd.lepenies@mpikg.mpg.de (BL)

Citation: Rabes A, Zimmermann S, Reppe K, Lang R, Seeberger PH, Suttorp N, et al. (2015) The C-Type Lectin Receptor Mincle Binds to Streptococcus pneumoniae but Plays a Limited Role in the AntiPneumococcal Innate Immune Response. PLoS ONE 10(2): e0117022. doi:10.1371/journal.pone.0117022

Academic Editor: Eliane Namie Miyaji, Instituto Butantan, BRAZIL

Received: September 23, 2014

Accepted: December 17, 2014

Published: February 6, 2015

Copyright: @ 2015 Rabes et al. This is an open access article distributed under the terms of the Creative Commons Attribution License, which permits unrestricted use, distribution, and reproduction in any medium, provided the original author and source are credited.

Data Availability Statement: All relevant data are within the paper.

Funding: This work was supported by the Deutsche Forschungsgemeinschaft (GRK1673/B5 to A.R., M. W. and B.O., OP 86/7-2 to M.W. and B.O., SFB/TR84 project $\mathrm{C} 3 / 6$ to M.W., project $\mathrm{B} 1$ to N.S., and project A1/A5 to B.O., SFB765 project B8 to P.H.S. and B.L., and SFB796 project B6 to R.L.). S.Z. and B.L. acknowledge funding by the IMPRS on Multiscale Bio-Systems. Funding by the German Federal Ministry of Education and Research (BMBF, Fkz. 0315446 to B.L. and $01 \mathrm{ZX} 1304$ B to M.W.) is also

\section{Abstract}

The innate immune system employs C-type lectin receptors (CLRs) to recognize carbohydrate structures on pathogens and self-antigens. The Macrophage-inducible C-type lectin (Mincle) is a FcRy-coupled CLR that was shown to bind to mycobacterial cord factor as well as certain fungal species. However, since CLR functions during bacterial infections have not yet been investigated thoroughly, we aimed to examine their function in Streptococcus pneumonia infection. Binding studies using a library of recombinantly expressed CLR-Fc fusion proteins indicated a specific, $\mathrm{Ca}^{2+}$-dependent, and serotype-specific binding of Mincle to $S$. pneumonia. Subsequent experiments with different Mincle-expressing cells as well as Mincle-deficient mice, however, revealed a limited role of this receptor in bacterial phagocytosis, neutrophil-mediated killing, cytokine production, and antibacterial immune response during pneumonia. Collectively, our results indicate that Mincle is able to recognize S. pneumonia but is not required for the anti-pneumococcal innate immune response.

\section{Introduction}

Streptococcus pneumoniae frequently colonizes the upper respiratory tract of humans. Depending on the immune status of the host, on preceding viral infections, and on the pneumococcal serotype, this asymptomatic colonization can progress to invasive diseases. These diseases, that include community-acquired pneumonia, sepsis, and meningitis, cause significant mortality especially in children and the elderly $[1,2]$. Important virulence factors of $S$. pneumoniae are 
gratefully acknowledged. The funders had no role in study design, data collection and analysis, decision to publish, or preparation of the manuscript.

Competing Interests: The authors have declared that no competing interests exist. the exotoxin pneumolysin (PLY) [3], and the polysaccharide capsule that inhibits phagocytosis, complement factor binding, and entrapment by neutrophil extracellular traps [4-6]. The innate immune system detects $S$. pneumoniae through pattern recognition receptors (PRRs) that belong to different protein families and functional classes $[7,8]$. For example, the Toll-like receptor (TLR) members TLR2 and TLR9 detect pneumococcal cell wall components and CpG-rich DNA, respectively [9-11]. Among NOD-like receptors (NLRs), NOD2 recognizes pneumococcal peptidoglycan and NLRP3 is activated by PLY [12-15]. Moreover, AIM2 as well as another STING-dependent cytosolic DNA sensor detect pneumococcal nucleic acid in the host cell cytosol $[7,12]$. These receptors primarily regulate the production of NF- $\mathrm{\kappa B}-$ dependent proinflammatory mediators, IL-1 family cytokines, and type I IFNs.

The myeloid C-type lectin receptors (CLRs) represent an additional family of sensors that recognize carbohydrates as well as other ligands of both pathogens and self [16-18]. The CLRs are transmembrane proteins that share a conserved protein fold, termed carbohydrate recognition domain (CRD). The CRD consists of two protein loops and two antiparallel $\beta$-sheets, stabilized by highly conserved disulfide bonds and up to four $\mathrm{Ca}^{2+}$-binding sites [19]. Thus, ligand binding by CLRs is often mediated in a $\mathrm{Ca}^{2+}$-dependent fashion. The cytoplasmic domains of CLRs frequently contain either hemITAM or ITIM signaling motifs, or associate with ITAM-bearing adaptors such as Fc receptor common $\gamma$ chain $(\mathrm{FcR} \gamma)$ and DAP12. Whereas hemITAM- and ITAM-mediated signaling stimulates myeloid cell activation through Syk, ITIM-containing CLRs recruit phosphatases and negatively regulate kinase-dependent signaling pathways [16]. While CLRs were shown to interact with a large number of fungi, viruses, or parasites, currently there is limited data available on the function of CLRs in bacterial recognition and the activation of anti-bacterial immune responses [20].

The CLR Macrophage-inducible C-type lectin (Mincle, Clec4e) is a type II transmembrane protein that contains a single CRD. Mincle is predominantly expressed by APCs of the myeloid lineage such as macrophages and DCs. The Mincle gene is located in the natural killer gene complex together with three related and highly conserved type II CLR genes (encoding MCL, DCIR and Dectin-2), found on murine chromosome 6 (human chromosome 12) [21, 22]. Mincle has been demonstrated to recognize the mycobacterial glycolipid trehalose-6, 6'-dimycolate (TDM, cord factor) [23-25]. Recently, the structural requirements for TDM binding by Mincle have been elucidated by crystallographic analyses [26-28]. In addition, Mincle recognizes Malassezia and Candida strains, as well as the endogenous ribonucleoprotein SAP130 [29-32]. Since Mincle does not itself express an intracellular signaling domain, it associates with FcR $\gamma$ chain to stimulate a Syk- and CARD9/Bcl10/Malt1-mediated cascade culminating in the production of NF- $\mathrm{KB}$-dependent proinflammatory cytokines [31, 33]. Fungal engagement of Mincle, however, has also been shown to suppress Dectin-1- and IRF1-mediated IL-12 production by activating the E3 ubiquitin ligase Mdm2 through Syk-CARD9-PI3K [34]. Moreover, Mincle contributes to neutrophil activation, phagocytosis, and bacterial killing upon M. tuberculosis and Klebsiella pneumoniae infection [35, 36].

In the present study, we used a library of recombinantly expressed CLR-Fc fusion proteins to analyze the contribution of CLRs to $S$. pneumoniae recognition. We identified Mincle as a CLR that bound to $S$. pneumoniae in a $\mathrm{Ca}^{2+}$-dependent manner. To analyze whether the Mincle/S. pneumoniae interaction impact the immune response, different primary cells and a murine S. pneumoniae infection model was employed. However, infection of Mincle- and FcR $\gamma$ deficient cells and mice indicated that Mincle did not influence the course of infection suggesting a limited role for Mincle in immunity against S. pneumoniae. 


\section{Materials and Methods}

\section{Bacterial strains}

S. pneumoniae serotype (ST)3 strain PN36 (NCTC7978), ST2 strains D39 and D39Acps, ST1 multilocus sequence type (MLST)306 strain and ST9N MLST66 strain were used. Bacteria were grown in THY media at $37^{\circ} \mathrm{C}$ and $5 \% \mathrm{CO}_{2}$ until they reached a phase of logarithmic growth. For heat-inactivation, bacteria where incubated at $56^{\circ} \mathrm{C}$ for $1 \mathrm{~h}$ while shaking. Trichosporon cutaneum was grown in YEPD medium at $26^{\circ} \mathrm{C}$ for $2-3$ days and was then heatinactivated at $80^{\circ} \mathrm{C}$ for $20 \mathrm{~min}$.

\section{Production of the CLR-Fc fusion protein library}

The library of CLR-Fc fusion proteins was prepared as described previously [37-39]. Briefly, murine splenic RNA was reverse transcribed into cDNA using Reverse Transcriptase (New England Biolabs, Ipswich, USA). The cDNA encoding the extracellular part of each CLR was amplified by polymerase chain reaction (PCR) and was then ligated into the pFuse-hIgG1-Fc expression vector (InvivoGen, San Diego, USA). The CLR-Fc vector constructs were either stably transfected into CHO cells or transiently transfected using the FreeStyle Max CHO-S Expression System (Life Technologies, Darmstadt, Germany). Purification of the CLR-Fc fusion proteins from the cell supernatant was performed using HiTrap Protein G HP columns (GE Healthcare, Piscataway, USA). The purity of each CLR-Fc fusion protein was confirmed by sodium dodecyl sulfate polyacrylamide gel electrophoresis (SDS-PAGE) and subsequent Coomassie stain, Western Blot using anti-human IgG-HRP antibody (Dianova, Hamburg, Germany) as well as mass spectrometry.

\section{Binding of Mincle-Fc to immobilized S. pneumoniae}

Heat inactivated bacteria diluted in PBS were coated on 96-well high binding plates (Greiner, Frickenhausen, Germany) at a concentration of $3 \times 10^{8}$ cells $/ \mathrm{mL}$ overnight. After blocking with $1 \% \mathrm{BSA}$ in PBS, $10 \mu \mathrm{g} / \mathrm{mL}$ of each CLR-Fc fusion protein was incubated in lectin binding buffer (50 mM HEPES, $5 \mathrm{mM} \mathrm{MgCl}_{2}, 5 \mathrm{mM} \mathrm{CaCl}_{2}$, pH 7.4) or EDTA buffer (10 mM EDTA, $50 \mathrm{mM}$ HEPES, pH 7.4) at RT for $2 \mathrm{~h}$. Binding of Mincle to plate-bound trehalose-6, 6'-dibehenate (TDB, $50 \mu \mathrm{g} / \mathrm{mL}$ ) was used as positive control. For competition experiments, heat-inactivated bacteria were pre-incubated with $10 \mu \mathrm{g} / \mathrm{mL}$ Mincle-Fc at $4^{\circ} \mathrm{C}$ for $15 \mathrm{~min}$. The binding of CLRFc fusion proteins was detected by an alkaline phosphatase-conjugated goat anti-hFc antibody (Dianova, Hamburg, Germany). Development was performed with p-nitrophenyl phosphate (Thermo Scientific, Rockford, IL, USA).

\section{Flow cytometric analysis of Mincle-Fc binding to S. pneumoniae}

Heat-inactivated bacteria were labeled with SYTO61 Red Fluorescent Nucleic Acid Stain (2.5 $\mu \mathrm{M}$ in PBS; Life Technologies) at RT for $30 \mathrm{~min}$ and were afterwards washed three times in PBS. To analyze the $\mathrm{Ca}^{2+}$ dependency of the interaction, $50 \mu \mathrm{L}$ labeled bacteria $\left(3 \times 10^{8}\right.$ cells $\left./ \mathrm{mL}\right)$ were incubated with $20 \mu \mathrm{g} / \mathrm{mL}$ Mincle-Fc diluted in lectin binding buffer or EDTA buffer at $4^{\circ} \mathrm{C}$ for $1 \mathrm{~h}$. After three washing steps with PBS, Mincle-Fc binding to S. pneumoniae was detected with a PE-conjugated goat anti-hFc antibody (Dianova, Hamburg, Germany). Flow cytometric analysis was performed with a FACSCanto II flow cytometer (BD Pharmingen, Heidelberg, Germany). Data were analyzed using the FlowJo analysis software (Tree Star Inc., Ashland, OR, USA). 


\section{Cells and infection}

Primary cells were isolated from wild-type (WT), Fcerg1 ${ }^{-1}$ (encoding FcR $\gamma$ ) [40] or Mincle ${ }^{-1}$ [29] mice on a C57Bl/6J background. Alveolar macrophages (AMФs) were obtained by bronchoalveolar lavage from mouse lungs. For isolation of alveolar epithelial cells (AECs), lung homogenates were prepared and leukocytes and endothelial cells were depleted from the cell suspension by incubation with biotinylated rat anti-mouse CD45, CD16/32 and CD31 (BD Pharmingen, Heidelberg, Germany) followed by magnetic separation. Microvascular endothelial cells (MVECs) were isolated from lung homogenates by positive magnetic selection using biotinylated rat anti-mouse CD144 (BD Pharmingen). For culturing, plates were coated with fibronectin. Bone marrow-derived macrophages (BMMs) were prepared from the bone marrow and cultured in RPMI 1640 containing 30\% L929 cell supernatant and 20\% FCS for 10 days. Polymorphonuclear leukocytes (PMNs) were isolated from the bone marrow using the antiLy6G MicroBead Kit (Miltenyi Biotech, Bergisch Gladbach, Germany). Cells were infected with S. pneumoniae ST2 (D39) or were stimulated with TDM (Sigma, St. Louis, USA) or LPS (Alexis Biochemicals, San Diego, USA).

\section{Bacterial uptake and killing assays}

BMMs were infected with S. pneumoniae ST2 (D39 with $50 \mathrm{mg} / \mathrm{mL}$ gentamicin. Intracellular, viable bacteria were determined $30 \mathrm{~min}$ or $90 \mathrm{~min}$ after treatment. Cells were PBS-washed and lysed with $1 \%$ saponin for $10 \mathrm{~min}$. Serial dilutions of the bacterial suspensions were plated on blood agar plates, and CFUs were determined. In PMNs, opsonophagocytic killing assays were conducted as described previously [41]. In brief, S. pneumoniae ST2 (D39 $\Delta c p s$ ) were pre-opsonized with infant rabbit serum (Pel-Freez Biologicals, Rogers, USA) at $37^{\circ} \mathrm{C}$ for $30 \mathrm{~min}$ before $\mathrm{PMN}$ were added. The percentage of viable bacteria was determined relative to control reactions lacking neutrophils after incubation at $37^{\circ} \mathrm{C}$ for $45 \mathrm{~min}$.

\section{Ethics statement}

Animal experiments were performed in strict accordance with the German regulations of the Society for Laboratory Animal Science and the European Health Law of the Federation of Laboratory Animal Science Associations. The protocol was approved by the Landesamt für Gesundheit und Soziales Berlin (Permit No. G 0210/11, G 0304/12, G 0357/12). All efforts were made to minimize suffering.

\section{Murine pneumonia model}

Female 8-10 weeks old WT, Fcer $1 g^{-/-}$and Mincle $^{-/-}$mice on C57Bl/6J background were housed in environmentally enriched and individually ventilated cages under specific pathogen free conditions. Food and water was given ad libitum. For infection, mice were anesthetized by i.p. ketamine $(80 \mathrm{mg} / \mathrm{kg})$ and xylazine $(25 \mathrm{mg} / \mathrm{kg})$ and transnasally inoculated with $7.5 \times 10^{4}$ or $5 \times 10^{6} \mathrm{CFU}$ S. pneumoniae ST3 (PN36) in $20 \mu \mathrm{L}$ PBS or sham-infected with $20 \mu \mathrm{L}$ PBS as described previously [13]. Mice were monitored every $12 \mathrm{~h}$ (more often if severely ill) and were humanely sacrificed by i.p. ketamine $(240 \mathrm{mg} / \mathrm{kg})$ and xylazine $(112,5 \mathrm{mg} / \mathrm{kg})$ when they reached the predefined humane endpoints (body temperature $<30^{\circ} \mathrm{C}$, body weight loss $>20 \%$ ). Survival was recorded for 10 days or mice were sacrificed $6,12,24$, or $48 \mathrm{~h}$ p.i. For analysis, anesthetized (160 mg/kg mg ketamine, $75 \mathrm{mg} / \mathrm{kg}$ xylazine) mice were heparinized, tracheotomised and ventilated and perfused via the pulmonary artery with saline for $2 \mathrm{~min}$. 


\section{Determination of bacterial load, cell recruitment and cytokines}

Bacterial loads were determined in the bronchoalveolar lavage fluid (BALF) and blood. Serial dilutions of samples were plated on blood agar and CFUs were determined. BAL cells were counted by haemocytometer and differentiated by flow cytometry (FACSCalibur; BD) as described previously [13]. Cytokines were quantified by ELISA in the BALF or by quantitative RT-PCR from total cellular RNA of the lung.

\section{ELISA}

Concentrations of IL-6, TNF $\alpha$ and $\mathrm{KC}$ in the BAL or in cell-free supernatants were quantified by commercially available sandwich ELISA kits (eBioscience, Frankfurt, Germany; R\&D, Minneapolis, USA).

\section{Quantitative RT-PCR analysis}

Total cellular RNA was isolated, transcribed to cDNA, and amplified by quantitative RT-PCR using Gene Expression Master Mix (Applied Biosystems, Foster City, USA). TaqMan Gene Expression Assays were purchased from Applied Biosystems.

\section{Data Analysis}

Data are expressed as mean \pm SEM. For CLR-Fc binding studies, statistical analysis was performed using unpaired Student's t-test. For murine $S$. pneumoniae infection experiments, analysis was performed using the log-rank test for survival, and the Kruskal-Wallis test followed by Dunn's multiple comparison test for comparison of more than two groups. Data analysis was performed using the Prism software (GraphPad Software, La Jolla, CA). For all statistical analyses, $\mathrm{p}$ values $<0.05$ were considered significant: $* \mathrm{p}<0.05, * * \mathrm{p}<0.01$, $* * * \mathrm{p}<0.001, * * * * \mathrm{p}<0.0001$.

\section{Results}

\section{Mincle binds to S. pneumoniae}

To analyze whether CLRs are involved in S. pneumoniae recognition, we performed an initial ELISA screening for CLR binding to heat-killed S. pneumoniae ST3 using a comprehensive library of CLR-Fc fusion proteins [37] (Fig. 1A). Binding of the murine DC-SIGN homolog Specific intercellular adhesion molecule-grabbing nonintegrin receptor 1 (SIGNR1) was used as a positive control since SIGNR1 on marginal zone macrophages was previously reported to be crucial for S. pneumoniae recognition [42]. Indeed, SIGNR1-Fc exhibited substantial binding to plate-bound S. pneumoniae (Fig. 1A). Besides SIGNR1-Fc, the ELISA-based pre-screening revealed Mincle as a candidate CLR that bound to heat-killed S. pneumoniae (Fig. 1A). In contrast, numerous other CLR-Fc fusion proteins, including MCL, DCAR, DCIR, CLEC-9a, MICL, CLEC-12b, SIGNR3, and MGL1 exhibited no or very weak binding to plate-bound S. pneumoniae. To confirm the specificity of the Mincle/S. pneumoniae ST3 interaction, we performed flow cytometric binding assays (Fig. 1B-C). In agreement with the ELISA-based binding assay, flow cytometric analysis indicated substantial binding of Mincle-Fc to S. pneumoniae ST3 (Fig. 1B). Furthermore, we performed comparative binding studies with MincleFc and other bacteria and fungi. In a previous study, the fungus T. cutaneum did not induce Mincle activation in a Mincle reporter cell line-based assay [32]. Indeed, whereas a high percentage of $S$. pneumoniae was recognized by Mincle-Fc, we observed only marginal binding of Mincle-Fc to T. cutaneum. These findings indicate that Mincle specifically recognizes S. pneumoniae. 
A

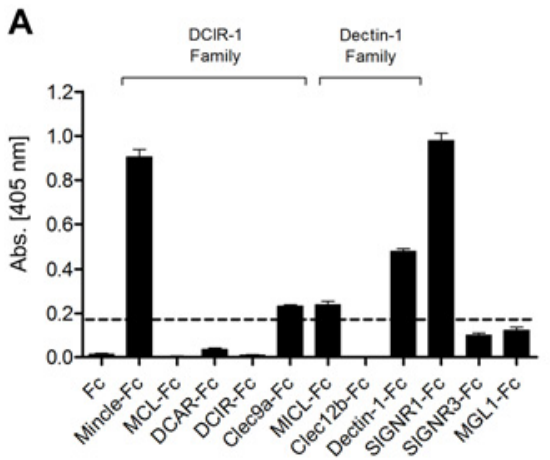

B

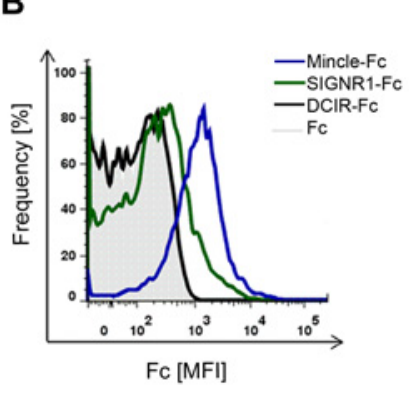

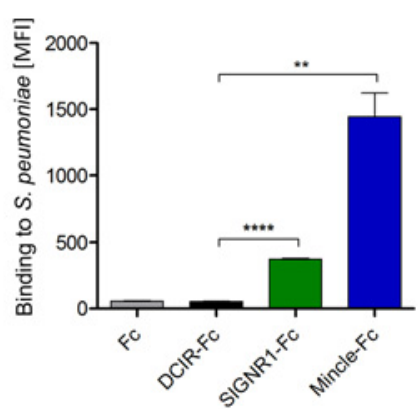

C

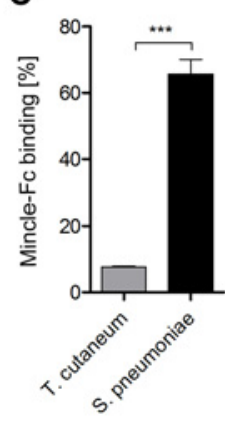

Figure 1. Mincle binds to S. pneumoniae. (A) Heat-inactivated S. pneumoniae ST3 $\left(1.5 \times 10^{7}\right.$ cells/well) diluted in PBS were immobilized on ELISA plates and incubated with CLR-Fc fusion proteins $(10 \mu \mathrm{g} / \mathrm{mL})$ diluted in lectin binding buffer. Data are representative of three independent experiments (triplicates each). The dashed line indicates background binding of CLR-Fc fusion proteins. (B) Binding of Mincle-Fc, SIGNR1-Fc and DCIR-Fc was analyzed by flow cytometry. CLR-Fc fusion proteins $(20 \mu \mathrm{g} / \mathrm{mL})$ were incubated with S. pneumoniae ST3 at a concentration of $3 \times 10^{8}$ cells $/ \mathrm{mL}$ diluted in lectin binding buffer. Left: Representative histogram plot of one binding experiment. Right: Statistical analysis of the flow cytometry-based binding assay. Data are representative of three independent experiments (triplicates each). (C) Mincle-Fc binding to heat-killed T. cutaneum and S. pneumoniae ST3 was analyzed by flow cytometry. Percentage of binding is shown relative to $\mathrm{Fc}$ binding to each pathogen. Data are representative of three independent experiments (triplicates each). (A-C), Data are shown as mean + SEM. Significance is indicated by asterisks, ${ }^{*}=p<0.05 ;{ }^{*}=p<0.01 ;{ }^{* *}=p<0.001 ;{ }^{* * *}=p<0.0001$.

doi:10.1371/journal.pone.0117022.g001

\section{The Mincle/S. pneumoniae interaction is $\mathrm{Ca}^{2+}$-dependent and serotype specific}

Next, we determined whether the Mincle-Fc binding to $S$. pneumoniae was mediated in a $\mathrm{Ca}^{2+}$-dependent manner. Previously, it was shown that Mincle binds to its known ligands TDM (cord factor) and TDB in a $\mathrm{Ca}^{2+}$ dependent fashion [24, 25]. Pre-incubation of Mincle$\mathrm{Fc}$ in a buffer containing the $\mathrm{Ca}^{2+}$ chelating agent EDTA resulted in reduced binding of Mincle-Fc to plate-bound TDB as well as S. pneumoniae ST3 suggesting a $\mathrm{Ca}^{2+}$-dependent Mincle/S. pneumoniae interaction (Fig. 2A). This finding was corroborated by flow cytometry since the Mincle-Fc incubation in an EDTA-containing buffer markedly reduced its binding to

A

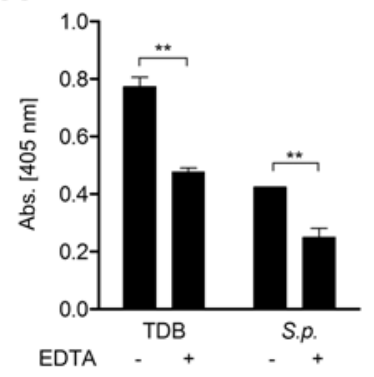

B

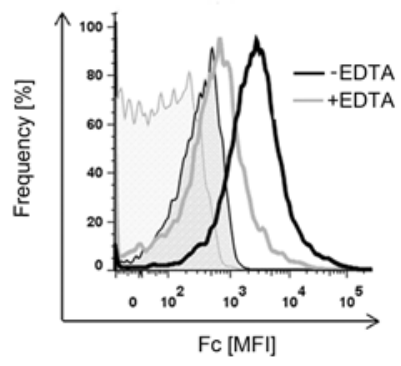

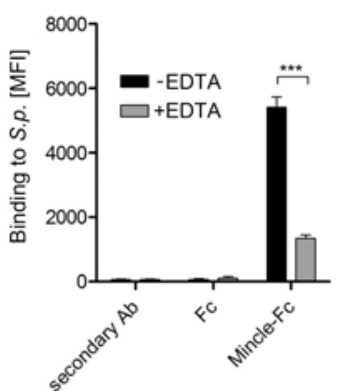

C

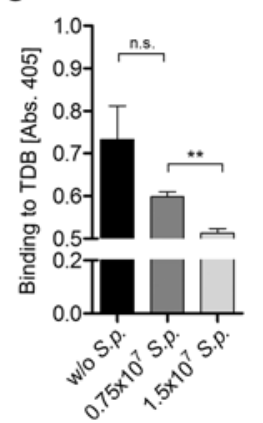

Figure 2. Mincle binding to S. pneumoniae is $\mathrm{Ca}^{2+}$-dependent. (A) To analyze the $\mathrm{Ca}^{2+}$ dependency of the Mincle/S. pneumoniae interaction, TDB $\left(50 \mu \mathrm{g} / \mathrm{mL}\right.$ in isopropanol) and heat-inactivated S. pneumoniae ST3 $\left(3 \times 10^{8}\right.$ cells $/ \mathrm{mL}$ in PBS) were immobilized on ELISA plates. Binding of Mincle-Fc $(10 \mu \mathrm{g} / \mathrm{mL})$ diluted in lectin binding buffer or EDTA buffer (10 mM EDTA) to immobilized TDB and S. pneumoniae ST3 was analyzed. Data are representative of three independent experiments (triplicates each). (B) Mincle-Fc $(20 \mu \mathrm{g} / \mathrm{mL}$ ) (non-filled curves) or Fc only (gray-filled curves) were incubated with S. pneumoniae ST3 $\left(3 \times 10^{8} \mathrm{cells} / \mathrm{mL}\right)$ in lectin binding buffer or EDTA buffer (10 mM EDTA). Left: Representative histogram plot of one binding experiment. Right: Statistical analysis of the flow cytometry-based binding assay in the presence or absence of EDTA. Data are representative of three independent experiments (triplicates each). (C) $20 \mu \mathrm{g} / \mathrm{mL}$ Mincle-Fc, diluted in lectin binding buffer, was pre-incubated with S. pneumoniae ST3 and subsequently incubated with plate-bound TDB $(50 \mu \mathrm{g} / \mathrm{mL})$. Data are representative of three independent experiments (triplicates each). (A-C), Data are shown as mean + SEM. Significance is indicated by asterisks, ${ }^{*}=p<0.05 ;{ }^{*}=p<0.01 ; * * *=p<0.001$. 
S. pneumoniae (Fig. 2B). Next, we performed an ELISA-based competition assay with increasing concentrations of heat-killed S. pneumoniae ST3 to disrupt the Mincle-Fc binding to the Mincle ligand TDB (Fig. 2C). Indeed, Mincle-Fc incubation in the presence of $S$. pneumoniae led to reduced binding to TDB, thus confirming the specificity of the Mincle/S. pneumoniae interaction. To analyze if Mincle binding to $S$. pneumoniae is serotype specific, we performed binding studies with different S. pneumoniae serotypes (ST1, ST2, ST3 and ST9N). Indeed, we observed a differential binding of Mincle-Fc to different serotypes. Mincle-Fc exhibited a strong binding to S. pneumoniae ST2 (D39) and ST3 (PN36), weaker binding to ST9N and almost no binding to ST1 (S1 Fig.). Thus, we conclude that Mincle-Fc binding to S. pneumoniae is serotype-specific suggesting a carbohydrate-specific binding of Mincle to S. pneumoniae.

\section{Mincle is not required for production of inflammatory cytokines, phagocytosis or bacterial killing upon S. pneumoniae infection}

Next, we examined the expression and function of Mincle in different cell types. We found that Mincle expression is low in alveolar epithelial cells and lung microvascular endothelial cells, and higher in alveolar macrophages, bone-marrow macrophages (BMMs) and neutrophils (Fig. 3A). Mincle expression was up-regulated upon pneumococcal infection in lung epithelial cells, endothelial cells and macrophages. Lack of Mincle or FcR $\gamma($ Fcerg1-/-) in alveolar macrophages and BMMs did not significantly affect the S. pneumoniae ST2-induced production of TNF $\alpha$ or KC (Fig. 3B-D). In contrast, cytokine production stimulated by TDM, which served as a positive control, was abolished in $\mathrm{Mincle}^{-/-}$and $\mathrm{Fcer}^{-g^{-/}}$cells cells (Fig. 3E). Moreover, phagocytosis and killing of $S$. pneumoniae by macrophages and neutrophils were not significantly affected by Mincle or FcR $\gamma$ deficiency (Fig. 3F-G). Thus, our data suggest that

$\mathbf{A}_{100000}$
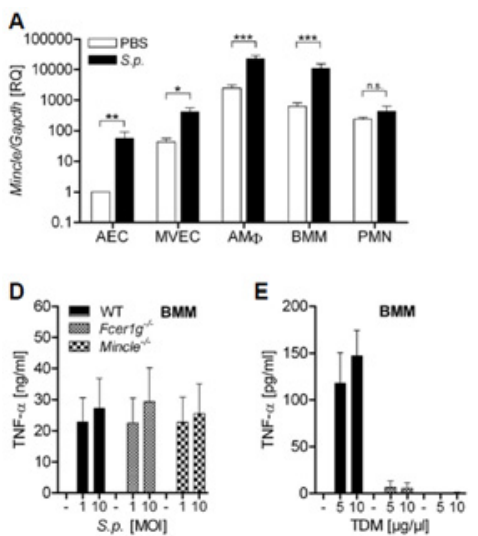

B
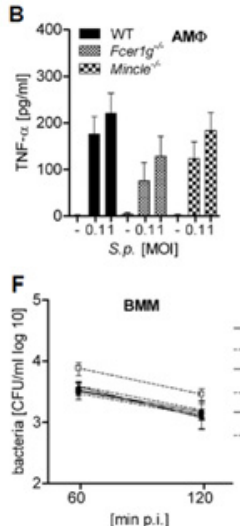

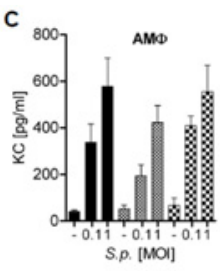

G

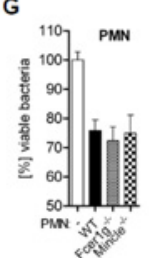

Figure 3. Lack of FcRy or Mincle does not affect the innate immune response to S. pneumoniae in different cell types. (A) AECs, MVECs and AMФs were isolated from the lung, and PMNs and BMMs from the bone marrow of C57BI/6 mice. Cells were left untreated or infected with S. pneumoniae ST2 (D39). (B-G) AMФs, BMMs and PMNs were isolated from WT, Fcer1 $\mathrm{g}^{-/-}$and Mincle $\mathrm{e}^{-/-}$mice. (B, C) AMФs or (D) BMMs were infected with S. pneumoniae ST2 or (E) BMMs were stimulated with TDM as a positive control for $16 \mathrm{~h}$ and cytokine release was quantified by ELISA. (F) Untreated or LPS-treated (100 ng/ml, $4 \mathrm{~h})$ BMMs were infected with $S$. pneumoniae ST2 $\Delta c p s(\mathrm{MOI}=2.5)$ and were treated with gentamicin $(50 \mathrm{mg} / \mathrm{ml})$ after $30 \mathrm{~min}$. Intracellular, viable bacteria were determined $60 \mathrm{~min}$ or $120 \mathrm{~min}$ post infection. (G) Rabbit serum-opsonized S. pneumoniae ST2 $\Delta c p s$ were incubated with PMNs and neutrophil-mediated killing was assessed after 45 min incubation. Data are shown as mean + SEM of (A) two (A: PMN, E, F), three $(C, G)$, four (A: AEC, MVEC, B, D) or five (A: AMФ, BMM) independent experiments carried out in duplicates (A-F) or quadruplicates $(G) ;{ }^{*}=p<0.05 ;{ }^{*}=p<0.01 ;{ }^{* * *}=p<0.001$; n.s. not significant.

doi:10.1371/journal.pone.0117022.g003 
S. pneumoniae recognition by Mincle and Mincle-dependent signaling might be dispensable for the innate immune response of macrophages and neutrophils towards $S$. pneumoniae.

\section{Mincle is not required for the antibacterial innate immune response during pneumococcal pneumonia}

Although Mincle signaling did not affect innate immune responses of different cell subsets to S. pneumoniae in vitro, a Mincle-dependent bacterial detection in other cell types, or alternatively Mincle-mediated responses to endogenous danger molecules such as SAP130 [31] could regulate the antibacterial immune response in vivo. We therefore examined the function of Mincle and its adapter molecule FcR $\gamma$ during pneumococcal pneumonia. Intranasal infection of wild-type mice with S. pneumoniae ST3 led to increased expression of Mincle in the whole lung (Fig. 4A). However, Mincle $e^{-/}$, Fcerlg $g^{-/-}$and wild-type mice did not significantly differ in their survival following S. pneumoniae infection (Fig. 4B). Moreover, mice lacking Mincle or its adapter molecule displayed unaltered bacterial loads in the bronchoalveolar lavage fluid (BALF) as compared to wild-type animals (Fig. 4C). To evaluate systemic dissemination of S. pneumoniae in these animals, bacterial loads in blood and spleen were determined. We detected high bacterial numbers in blood and spleens of all groups of infected mice $48 \mathrm{~h}$ p.i. (Fig. 4D and data not shown). According to the unaltered bacterial loads, S. pneumoniaeinfected Mincle $^{-/}$, Fcer $g^{-/-}$and wild-type mice exhibited a similar recruitment of neutrophils
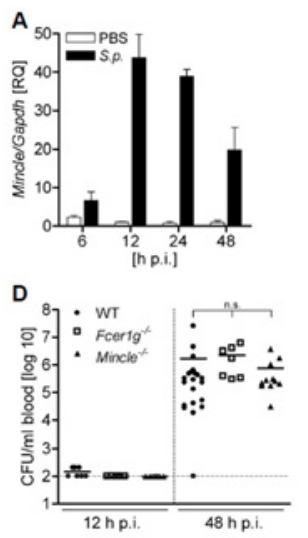

B
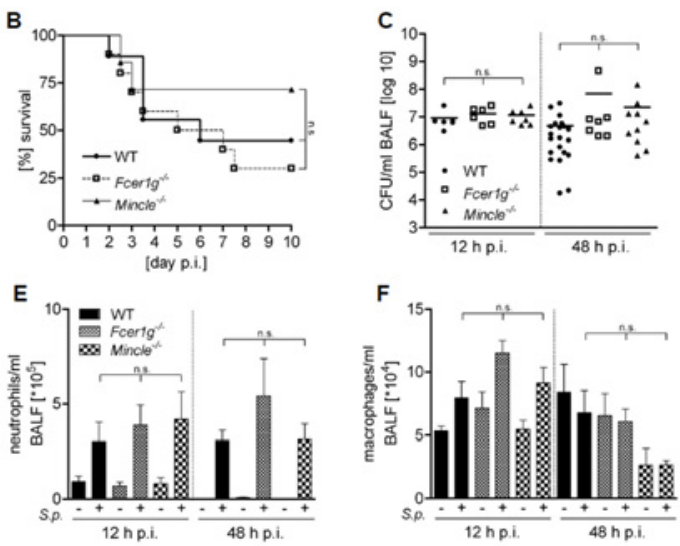
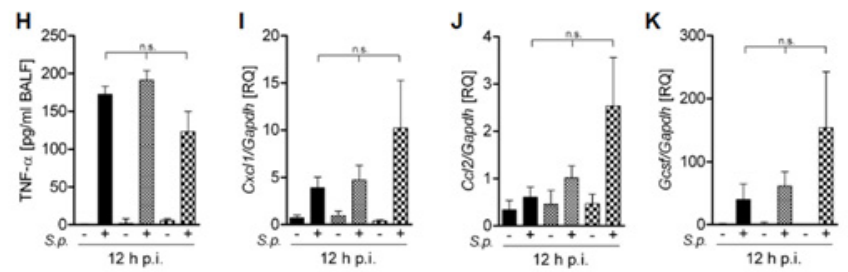

Figure 4. Lack of Mincle or FcRy does not influence the antibacterial host response during pneumococcal pneumonia. (A) C57BI/6 mice were intranasally infected with $5 \times 10^{6} \mathrm{CFU} /$ mouse S. pneumoniae ST3 or control treated with PBS and Mincle expression levels in the lungs at the indicated time points were determined by quantitative RT-PCR. (B) WT, Fcerg $1^{-/-}$and $M i n c l e^{-/-}$mice were intranasally infected with $7.5 \times 10^{4} \mathrm{CFU} /$ mouse S. pneumoniae ST3 and survival was monitored every $12 \mathrm{~h}$ over 10 days. (C-K) WT, Fcer1 $\mathrm{g}^{-/-}$and Mincle $^{-/-}$mice were intranasally infected with $5 \times 10^{6} \mathrm{CFU} /$ mouse S. pneumoniae ST3 or treated with PBS. Bacterial loads were determined in the (C) bronchoalveolar lavage fluid (BALF) and (D) blood. (E) Neutrophils and (F) macrophages in the BALF were quantified by flow cytometry. (G) IL-6 and (H) TNFa $(\mathrm{H})$ levels in the BALF were analyzed by ELISA. Relative expression of (I) Cxc/1, (J) CCl2 and (K) Gcsf in the lung was determined by quantitative RT-PCR. Data are shown as mean + SEM; $(A) n=3$, (B) $n=8-10,(C-F) n=6-22,(G-H) n=6-8,(I-K) n=4-5$ mice each group. n.s. not significant.

doi:10.1371/journal.pone.0117022.g004 
and macrophages to the lung (Fig. 4E-F). In addition, S. pneumoniae-induced production of cytokines and chemokines, many of which have been previously associated with Mincle activation in different models $[24,31]$, were not significantly affected by lack of Mincle or FcR $\gamma$ (Fig. 4G-K). These data collectively indicate that Mincle signaling is not required for antibacterial innate immune responses and resistance to $S$. pneumoniae ST3 in vivo.

\section{Discussion}

Myeloid CLRs are an important class of PRRs expressed by various immune cells and capable of detecting a broad spectrum of microbial and endogenous ligands. However, their function in acute bacterial infection has not been investigated systematically. For $S$. pneumoniae infection, SIGNR1 is one of the few CLRs shown to be crucial for bacterial recognition, phagocytosis as well as antibacterial defense $[43,44]$. We therefore set out to identify additional CLRs that are able to bind to S. pneumoniae. To this end, we used a comprehensive library of CLR-Fc fusion proteins covering immunologically relevant members of the Dectin-1 and DCIR family [37]. CLR-Fc fusion proteins are useful tools to identify pathogen/CLR interactions as they display the CRD in a dimeric fashion. Dimeric display allows for multivalent ligand binding and has helped to unravel novel CLR interactions with microbes or endogenous ligands [45, 46]. Out of our CLR-Fc library, we identified Mincle as a novel binder of S. pneumoniae, in addition to the known pneumococcal recognition by SIGNR1. We demonstrate that the Mincle/S. pneumoniae interaction occurs in a $\mathrm{Ca}^{2+}$-dependent fashion, as has been previously shown for the Mincle ligand TDM and pathogenic fungi [24, 30, 32].

To determine whether the Mincle/S. pneumoniae interaction impacts the innate immune response, we employed various primary immune cells and the murine infection model of pneumococcal pneumonia. However, we did not observe a functional consequence of this interaction for $S$. pneumoniae-induced innate responses in vitro and in vivo. Our finding that Mincle and FcR $\gamma$-deficiencies did not affect cytokine production and phagocytosis in macrophages suggests that binding of S. pneumoniae to Mincle does not correlate with down-stream signaling. Alternatively, the loss of Mincle signaling in macrophages, neutrophils and mice might have been compensated by other functionally redundant CLRs not coupled to FcR $\gamma$, as commonly observed in different infection models using CLR-deficient cell and mouse lines [16]. In addition, PRRs such as the TLRs and NLRs known to be essential for antibacterial immunity against $S$. pneumoniae $[2,47]$ might have overcome potential Mincle-mediated effects. It appears reasonable that the recognition of prokaryote-specific PAMPs such as peptidoglycan and pore-forming toxins by those receptors might dominate the innate immune response to bacteria. In contrast, the recognition of broadly expressed carbohydrate ligands might play a more important and non-redundant role in infections with eukaryotic pathogens such as fungi and parasites $[20,48]$.

Mincle is known to recognize different glycolipids in M. tuberculosis and fungi [24, 27, 30]. Given that glycolipids such as lipoteichoic acid are also present in S. pneumoniae [49, 50], it is reasonable to speculate that those structures mediate the binding to Mincle. In addition, Mincle also binds to the endogenous protein SAP130, indicating that besides glycolipids nonglycosylated ligands may be recognized by Mincle as well [31]. This renders the recognition of a protein ligand present in $S$. pneumoniae possible, although it appears unlikely since all binding studies were performed using pneumococci after heat inactivation. The specificity of the S. pneumoniae/Mincle interaction was proven by different observations. First, we detected no or only marginal binding of most other CLR-Fc fusion proteins of the library to the bacterium, second the interaction was $\mathrm{Ca}^{2+}$-dependent, and third, it could be competitively inhibited. Considering this specific interaction, it may be interesting to elucidate in future studies whether 
combined deficiencies in Mincle and other CLRs and/or cross-talk mechanisms between Mincle and other PRRs act in a synergistic manner.

\section{Supporting Information}

S1 Fig. Binding of Mincle to S. pneumoniae is serotype-specific. Binding of Mincle-Fc to different $S$. pneumoniae serotypes was analyzed by flow cytometry. Mincle-Fc and hFc $(20 \mu \mathrm{g} /$ $\mathrm{mL}$ ) were incubated with S. pneumoniae serotype 1, serotype 2 (D39), serotype 3 (PN36), and serotype $9 \mathrm{~N}$ at a concentration of $3 \times 10^{8}$ cells $/ \mathrm{mL}$ in lectin binding buffer. Bound fusion protein was detected by a PE-conjugated goat anti-hFc antibody. Results are shown as MFI values $($ mean $+\mathrm{SEM})$ and are representative of three independent experiments (triplicates each). Significance is indicated by asterisks, $*=\mathrm{p}<0.05 ; * *=\mathrm{p}<0.01 ; * * *=\mathrm{p}<0.001 ; * * * *=\mathrm{p}<0.0001$; ns $=$ non-significant.

\section{Acknowledgments}

We are grateful to Maria Spelling, Dr. Maha Maglinao, Dr. Magdalena Eriksson, Timo Johannssen, and Theresa Wagner for technical assistance and reagent supply. We also thank Prof. Dr. Vera Meyer (Department of Applied and Molecular Microbiology, Technical University Berlin) for providing T. cutaneum.

\section{Author Contributions}

Conceived and designed the experiments: AR SZ BL BO. Performed the experiments: AR SZ KR BL. Analyzed the data: AR SZ BL BO. Contributed reagents/materials/analysis tools: RL KR PHS NS MW. Wrote the paper: AR SZ BL BO. Responsible principal investigators: BL BO.

\section{References}

1. Kadioglu A, Weiser JN, Paton JC, Andrew PW (2008) The role of Streptococcus pneumoniae virulence factors in host respiratory colonization and disease. Nat Rev Microbiol 6:288-301. doi: 10.1038/ nrmicro1871 PMID: 18340341

2. van der Poll T, Opal SM (2009) Pathogenesis, treatment, and prevention of pneumococcal pneumonia. Lancet 374: 1543-1556. doi: 10.1016/S0140-6736(09)61114-4 PMID: 19880020

3. Mitchell AM, Mitchell TJ (2010) Streptococcus pneumoniae: virulence factors and variation. Clin Microbiol Infect 16: 411-418. doi: 10.1111/j.1469-0691.2010.03183.x PMID: 20132250

4. Abeyta M, Hardy GG, Yother J (2003) Genetic alteration of capsule type but not PspA type affects accessibility of surface-bound complement and surface antigens of Streptococcus pneumoniae. Infect Immun 71: 218-225. doi: 10.1128/IAI.71.1.218-225.2003 PMID: 12496169

5. Hyams C, Camberlein E, Cohen JM, Bax K, Brown JS (2010) The Streptococcus pneumoniae capsule inhibits complement activity and neutrophil phagocytosis by multiple mechanisms. Infect Immun 78: 704-715. doi: 10.1128/IAI.00881-09 PMID: 19948837

6. Wartha F, Beiter K, Albiger B, Fernebro J, Zychlinsky A, et al. (2007) Capsule and D-alanylated lipoteichoic acids protect Streptococcus pneumoniae against neutrophil extracellular traps. Cell Microbiol 9: 1162-1171. doi: 10.1111/j.1462-5822.2006.00857.x PMID: 17217430

7. Koppe U, Hogner K, Doehn JM, Muller HC, Witzenrath M, et al. (2012) Streptococcus pneumoniae stimulates a STING- and IFN regulatory factor 3-dependent type I IFN production in macrophages, which regulates RANTES production in macrophages, cocultured alveolar epithelial cells, and mouse lungs. J Immunol 188: 811-817. doi: 10.4049/jimmunol.1004143 PMID: 22156592

8. Opitz B, van Laak V, Eitel J, Suttorp N (2010) Innate immune recognition in infectious and noninfectious diseases of the lung. Am J Respir Crit Care Med 181: 1294-1309. doi: 10.1164/rccm.200909-1427SO PMID: 20167850

9. Yoshimura A, Lien E, Ingalls RR, Tuomanen E, Dziarski R, et al. (1999) Cutting edge: recognition of Gram-positive bacterial cell wall components by the innate immune system occurs via Toll-like receptor 2. J Immunol 163: 1-5. PMID: 10384090 
10. Knapp S, Wieland CW, van 't Veer C, Takeuchi O, Akira S, et al. (2004) Toll-like receptor 2 plays a role in the early inflammatory response to murine pneumococcal pneumonia but does not contribute to antibacterial defense. J Immunol 172: 3132-3138. doi: 10.4049/jimmunol.172.5.3132 PMID: 14978119

11. Albiger B, Dahlberg S, Sandgren A, Wartha F, Beiter K, et al. (2007) Toll-like receptor 9 acts at an early stage in host defence against pneumococcal infection. Cell Microbiol 9: 633-644. doi: 10.1111/j.14625822.2006.00814.x PMID: 17004992

12. Fang R, Tsuchiya $K$, Kawamura I, Shen $Y$, Hara H, et al. (2011) Critical roles of ASC inflammasomes in caspase-1 activation and host innate resistance to Streptococcus pneumoniae infection. J Immunol 187: 4890-4899. doi: 10.4049/jimmunol.1100381 PMID: 21957143

13. Witzenrath M, Pache F, Lorenz D, Koppe U, Gutbier B, et al. (2011) The NLRP3 inflammasome is differentially activated by pneumolysin variants and contributes to host defense in pneumococcal pneumonia. J Immunol 187: 434-440. doi: 10.4049/jimmunol.1003143 PMID: 21646297

14. Opitz B, Puschel A, Schmeck B, Hocke AC, Rosseau S, et al. (2004) Nucleotide-binding oligomerization domain proteins are innate immune receptors for internalized Streptococcus pneumoniae. J Biol Chem 279: 36426-36432. doi: 10.1074/jbc.M403861200 PMID: 15215247

15. Davis KM, Nakamura S, Weiser JN (2011) Nod2 sensing of lysozyme-digested peptidoglycan promotes macrophage recruitment and clearance of S. pneumoniae colonization in mice. J Clin Invest 121: 3666-3676. doi: 10.1172/JCI57761 PMID: 21841315

16. Sancho D, Reis e Sousa $C$ (2012) Signaling by myeloid C-type lectin receptors in immunity and homeostasis. Annu Rev Immunol 30: 491-529. doi: 10.1146/annurev-immunol-031210-101352 PMID: 22224766

17. Kerscher B, Willment JA, Brown GD (2013) The Dectin-2 family of C-type lectin-like receptors: an update. Int Immunol 25: 271-277. doi: 10.1093/intimm/dxt006 PMID: 23606632

18. Lepenies B, Lee J, Sonkaria S (2013) Targeting C-type lectin receptors with multivalent carbohydrate ligands. Adv Drug Deliv Rev 65: 1271-1281. doi: 10.1016/j.addr.2013.05.007 PMID: 23727341

19. Drickamer K (1999) C-type lectin-like domains. Curr Opin Struct Biol 9: 585-590. doi: 10.1016/S0959440X(99)00009-3 PMID: 10508765

20. Hoving JC, Wilson GJ, Brown GD (2014) Signalling C-type lectin receptors, microbial recognition and immunity. Cell Microbiol 16: 185-194. doi: 10.1111/cmi.12249 PMID: 24330199

21. Flornes LM, Bryceson YT, Spurkland A, Lorentzen JC, Dissen E, et al. (2004) Identification of lectin-like receptors expressed by antigen presenting cells and neutrophils and their mapping to a novel gene complex. Immunogenetics 56: 506-517. doi: 10.1007/s00251-004-0714-x PMID: 15368084

22. Balch SG, Greaves DR, Gordon S, McKnight AJ (2002) Organization of the mouse macrophage C-type lectin (Mcl) gene and identification of a subgroup of related lectin molecules. Eur J Immunogenet 29: 61-64. doi: 10.1046/j.1365-2370.2002.00266.x PMID: 11841491

23. Matsunaga I, Moody DB (2009) Mincle is a long sought receptor for mycobacterial cord factor. J Exp Med 206: 2865-2868. doi: 10.1084/jem.20092533 PMID: 20008525

24. Schoenen H, Bodendorfer B, Hitchens K, Manzanero S, Werninghaus K, et al. (2010) Cutting edge: Mincle is essential for recognition and adjuvanticity of the mycobacterial cord factor and its synthetic analog trehalose-dibehenate. J Immunol 184: 2756-2760. doi: 10.4049/jimmunol.0904013 PMID: 20164423

25. Ishikawa E, Ishikawa T, Morita YS, Toyonaga K, Yamada H, et al. (2009) Direct recognition of the mycobacterial glycolipid, trehalose dimycolate, by C-type lectin Mincle. J Exp Med 206: 2879-2888. doi: 10.1084/jem.20091750 PMID: 20008526

26. Feinberg H, Jegouzo SA, Rowntree TJ, Guan Y, Brash MA, et al. (2013) Mechanism for recognition of an unusual mycobacterial glycolipid by the macrophage receptor mincle. J Biol Chem 288: 28457-28465. doi: 10.1074/jbc.M113.497149 PMID: 23960080

27. Furukawa A, Kamishikiryo J, Mori D, Toyonaga K, Okabe Y, et al. (2013) Structural analysis for glycolipid recognition by the C-type lectins Mincle and MCL. Proc Natl Acad Sci U S A 110: 17438-17443. doi: 10.1073/pnas.1312649110 PMID: 24101491

28. Jegouzo SA, Harding EC, Acton O, Rex MJ, Fadden AJ, et al. (2014) Defining the conformation of human mincle that interacts with mycobacterial trehalose dimycolate. Glycobiology. doi: 10.1093/ glycob/cwu072 PMID: 25028392

29. Wells CA, Salvage-Jones JA, Li X, Hitchens K, Butcher S, et al. (2008) The macrophage-inducible C-type lectin, mincle, is an essential component of the innate immune response to Candida albicans. $J$ Immunol 180: 7404-7413. doi: 10.4049/jimmunol.180.11.7404 PMID: 18490740

30. Bugarcic A, Hitchens K, Beckhouse AG, Wells CA, Ashman RB, et al. (2008) Human and mouse macrophage-inducible C-type lectin (Mincle) bind Candida albicans. Glycobiology 18: 679-685. doi: 10. 1093/glycob/cwn046 PMID: 18509109 
31. Yamasaki S, Ishikawa E, Sakuma M, Hara H, Ogata K, et al. (2008) Mincle is an ITAM-coupled activating receptor that senses damaged cells. Nat Immunol 9: 1179-1188. doi: 10.1038/ni.1651 PMID: 18776906

32. Yamasaki S, Matsumoto M, Takeuchi O, Matsuzawa T, Ishikawa E, et al. (2009) C-type lectin Mincle is an activating receptor for pathogenic fungus, Malassezia. Proc Natl Acad Sci U S A 106: 1897-1902. doi: 10.1073/pnas.0805177106 PMID: 19171887

33. Werninghaus K, Babiak A, Gross O, Holscher C, Dietrich H, et al. (2009) Adjuvanticity of a synthetic cord factor analogue for subunit Mycobacterium tuberculosis vaccination requires FcRgamma-SykCard9-dependent innate immune activation. J Exp Med 206: 89-97. doi: 10.1084/jem.20081445 PMID: 19139169

34. Wevers BA, Kaptein TM, Zijlstra-Willems EM, Theelen B, Boekhout T, et al. (2014) Fungal engagement of the C-type lectin mincle suppresses dectin-1-induced antifungal immunity. Cell Host Microbe 15: 494-505. doi: 10.1016/j.chom.2014.03.008 PMID: 24721577

35. Sharma A, Steichen AL, Jondle CN, Mishra BB, Sharma J (2014) Protective role of Mincle in bacterial pneumonia by regulation of neutrophil mediated phagocytosis and extracellular trap formation. $\mathrm{J}$ Infect Dis 209: 1837-1846. doi: 10.1093/infdis/jit820 PMID: 24353272

36. Lee WB, Kang JS, Yan JJ, Lee MS, Jeon BY, et al. (2012) Neutrophils Promote Mycobacterial Trehalose Dimycolate-Induced Lung Inflammation via the Mincle Pathway. PLoS Pathog 8: e1002614. doi: 10.1371/journal.ppat.1002614 PMID: 22496642

37. Maglinao M, Eriksson M, Schlegel MK, Zimmermann S, Johannssen T, et al. (2014) A platform to screen for C-type lectin receptor-binding carbohydrates and their potential for cell-specific targeting and immune modulation. J Control Release 175: 36-42. doi: 10.1016/j.jconrel.2013.12.011 PMID: 24368301

38. Eriksson M, Serna S, Maglinao M, Schlegel MK, Seeberger PH, et al. (2014) Biological evaluation of multivalent lewis X-MGL-1 interactions. Chembiochem 15: 844-851. doi: 10.1002/cbic.201490017 PMID: 24616167

39. Schlegel MK, Hutter J, Eriksson M, Lepenies B, Seeberger PH (2011) Defined presentation of carbohydrates on a duplex DNA scaffold. Chembiochem 12: 2791-2800. doi: 10.1002/cbic.201100511 PMID: 22052782

40. Takai T, Li M, Sylvestre D, Clynes R, Ravetch JV (1994) FcR gamma chain deletion results in pleiotrophic effector cell defects. Cell 76: 519-529. doi: 10.1016/0092-8674(94)90115-5 PMID: 8313472

41. Standish AJ, Weiser JN (2009) Human neutrophils kill Streptococcus pneumoniae via serine proteases. J Immunol 183: 2602-2609. doi: 10.4049/jimmunol.0900688 PMID: 19620298

42. Koppel EA, Wieland CW, van den Berg VC, Litjens M, Florquin S, et al. (2005) Specific ICAM-3 grabbing nonintegrin-related 1 (SIGNR1) expressed by marginal zone macrophages is essential for defense against pulmonary Streptococcus pneumoniae infection. Eur J Immunol 35: 2962-2969. doi: 10.1002/ eji.200526216 PMID: 16134084

43. Kang YS, Kim JY, Bruening SA, Pack M, Charalambous A, et al. (2004) The C-type lectin SIGN-R1 mediates uptake of the capsular polysaccharide of Streptococcus pneumoniae in the marginal zone of mouse spleen. Proc Natl Acad Sci U S A 101: 215-220. doi: 10.1073/pnas.0307124101 PMID: 14694198

44. Lanoue A, Clatworthy MR, Smith P, Green S, Townsend MJ, et al. (2004) SIGN-R1 contributes to protection against lethal pneumococcal infection in mice. J Exp Med 200: 1383-1393. doi: 10.1084/jem. 20040795 PMID: 15583012

45. Hutter J, Eriksson M, Johannssen T, Klopfleisch R, von Smolinski D, et al. (2014) Role of the C-Type Lectin Receptors MCL and DCIR in Experimental Colitis. PLoS One 9: e103281. doi: 10.1371/journal. pone.0103281 PMID: 25068517

46. Neumann K, Castineiras-Vilarino M, Hockendorf U, Hannesschlager N, Lemeer S, et al. (2014) Clec12a is an inhibitory receptor for uric acid crystals that regulates inflammation in response to cell death. Immunity 40: 389-399. doi: 10.1016/j.immuni.2013.12.015 PMID: 24631154

47. Koppe U, Suttorp N, Opitz B (2012) Recognition of Streptococcus pneumoniae by the innate immune system. Cell Microbiol 14: 460-466. doi: 10.1111/j.1462-5822.2011.01746.x PMID: 22212419

48. Maglinao M, Klopfleisch R, Seeberger PH, Lepenies B (2013) The C-type lectin receptor DCIR is crucial for the development of experimental cerebral malaria. J Immunol 191: 2551-2559. doi: 10.4049/ jimmunol.1203451 PMID: 23918990

49. Gisch N, Kohler T, Ulmer AJ, Muthing J, Pribyl T, et al. (2013) Structural reevaluation of Streptococcus pneumoniae Lipoteichoic acid and new insights into its immunostimulatory potency. J Biol Chem 288: 15654-15667. doi: 10.1074/jbc.M112.446963 PMID: 23603911

50. Schroder NW, Morath S, Alexander C, Hamann L, Hartung T, et al. (2003) Lipoteichoic acid (LTA) of Streptococcus pneumoniae and Staphylococcus aureus activates immune cells via Toll-like receptor (TLR)-2, lipopolysaccharide-binding protein (LBP), and CD14, whereas TLR-4 and MD-2 are not involved. J Biol Chem 278: 15587-15594. doi: 10.1074/jbc.M212829200 PMID: 12594207 